\title{
Nursing interventions in depressed children with low serum levels of BDNF
}

\author{
QINGRONG SUN ${ }^{1}$, CHUANYING CUI $^{1}$, YANXIA FU $^{1}$, SHUMEI MA $^{2}$ and HONGXIA LI ${ }^{3}$ \\ Departments of ${ }^{1}$ Pediatrics, ${ }^{2}$ Cardiovascular Medicine and ${ }^{3}$ Obstetrics, Binzhou City Center Hospital, \\ Binzhou, Shandong 251700, P.R. China
}

Received May 26, 2017; Accepted August 7, 2017

DOI: $10.3892 /$ etm.2017.4921

\begin{abstract}
The purpose of this study was to investigate the correlation between brain-derived neurotrophic factor (BDNF) in serum and depression in children, and explore the effects of different nursing protocols on patients with low levels of BDNF. We recruited 128 children with depression and 50 healthy subjects. Compared with healthy controls, the mRNA and protein levels of $B D N F$ in serum were lower in children with depression $(\mathrm{p}<0.01)$. We selected 60 depressed children with low BDNF and randomly divided them in two groups: comprehensive nursing $(\mathrm{n}=30)$ and regular nursing $(n=30)$. Compared to healthy children, there was a significant increase in Hamilton depression (HAMD) scores in patients with depression in childhood $(\mathrm{p}<0.01)$. After treatment, BDNF protein expression was higher in the comprehensive nursing group than that in the regular nursing group $(\mathrm{p}<0.05)$. Also, the HAMD score in the comprehensive nursing group was significantly lower than that in the regular nursing group $(\mathrm{p}<0.05)$. Compliance to treatment and quality of life after treatment improved in the comprehensive nursing group compared with the regular nursing group $(\mathrm{p}<0.05)$. Overall, a decrease in BDNF expression is closely correlated with depression, and comprehensive nursing care can significantly ameliorate the depression symptoms in pediatric patients, increase the BDNF expression, and improve compliance and quality of life. These results provide theoretical and practical significance for clinical nursing care of patients with depression in childhood.
\end{abstract}

\section{Introduction}

Depression is a common psychiatric disease characterized by abnormal mental state. Typical symptoms include impaired thinking and eccentric personality. In recent years, an increase

Correspondence to: Dr Qingrong Sun, Department of Pediatrics, Binzhou City Center Hospital, 108 Huanchengnan Road, Huimin, Binzhou, Shandong 251700, P.R. China

E-mail: drqingrongsun@163.com

Key words: depression, comprehensive nursing care, brain-derived neurotrophic factor in social pressure has been associated to an increase in depression $(1,2)$. The younger tendency in the presentation of depression has also generated an increase in pediatric patients with depression. To ameliorate the depression symptoms of pediatric patients, a diversified set of treatment strategies has been developed (3-5). Deficiency or functional disorder in brain-derived neurotrophic factor (BDNF) is closely associated with depression, and increasing the level and recovering the function of BDNF has clinical application against depression. BDNF functions downstream of cAMP/PKA/CREB pathways and plays critical roles in recognition, depression and neurodegenerative disorders $(6,7)$. Nursing care can have an impact on the treatment of depression. Evidence suggests that appropriate nursing strategies can help patients with depression to overcome the psychiatric obstacles and increase their quality of life (8-10). In this study, we analyzed the nursing effect on pediatric depression patients with low expression of BDNF and found that nursing care can significantly ameliorate depression.

\section{Subjects and methods}

Study subjects. We enrolled 128 pediatric patients with depression who were treated in Binzhou City Center Hospital between February, 2014 and February, 2016. Patients were aged between 7 and 16 years. The cohort had 55 males and 73 females. All pediatric patients were diagnosed with depression by experts specializing in diagnosis and treatment. Fifty healthy children were enrolled into the control group, with ages between 6 and 15 years, and containing 25 males and 25 females. All enrolled subjects were cleared of other chronic diseases. This study was approved by the Ethics Committee of Binzhou City Center Hospital. Signed written informed consents were obtained from the guardians. The research protocol was approved by the Ethics Committee of the hospital. All the patients received follow-up care for one year by the hospital. Accurate clinical history, pathological material, and treatment protocol were conserved through the study.

Semi-quantitative PCR. After total RNA was purified from peripheral blood from pediatric patients and healthy children using the TRIzol kit (Invitrogen, Garlsbad, CA, USA), the integrity of RNA was confirmed by agarose gel electrophoresis. The electrophoresis revealed that $28 \mathrm{~S}, 18 \mathrm{~S}$ and $5 \mathrm{~S}$ bands were 
clear, and the 28S band was twice as strong as the $5 \mathrm{~S}$ band, suggesting that the RNA was intact for the following experiments. cDNA was prepared by reverse transcription using the reverse transcription kit (Invitrogen). Using glyceraldehyde 3-phosphate dehydrogenase $(G A P D H)$ as internal reference, we performed semi-quantitative PCR (ABI, Foster City, CA, USA) to detect the mRNA levels of $B D N F$. The reaction conditions were: initial denaturation at $95^{\circ} \mathrm{C}$ for $5 \mathrm{~min} ; 95^{\circ} \mathrm{C}$ for $30 \mathrm{sec}, 64^{\circ} \mathrm{C}$ for $25 \mathrm{sec}$ and $72^{\circ} \mathrm{C}$ for $30 \mathrm{sec}(35$ cycles). Extension at $72^{\circ} \mathrm{C}$ for $5 \mathrm{~min}$. Primers were synthesized by Tiangen Biotech (Beijing, China) and the sequences are shown in Table I. At the end of the reaction, the results were observed in ultraviolet imaging system (Biometra, Göttingen, Germany) after agarose gel electrophoresis.

Western blot analysis. Peripheral blood samples from patients and healthy subjects was concentrated, lysed in radioimmunoprecipitation assay (RIPA) in a 1:1 ratio (volume:volume), and centrifuged at $8,500 \mathrm{x}$ g for $5 \mathrm{~min}$. Then, supernatant was removed and the pellet contained the total protein that was quantified using the bicinchoninic acid (BCA) assay kit. The total protein was separated using sodium dodecyl sulfate polyacrylamide gel electrophoresis (SDS-PAGE). We loaded $10 \mu \mathrm{l}$ of sample per well for electrophoresis at $80 \mathrm{~V}$ followed by transfer onto the polyvinylidene fluoride (PVDF) membrane at $100 \mathrm{~V}$ for $90 \mathrm{~min}$. The membranes were blocked for $2 \mathrm{~h}$ and the relevant bands were cut for incubation using rabbit anti-BDNF and anti- $\beta$-catenin antibodies (Cell Signaling Technology, Beverly, MA, USA) at $4^{\circ} \mathrm{C}$ overnight. Membranes were washed using Tris-buffered saline and Tween-20 (TBST) 3 times for $5 \mathrm{~min}$. Secondary antibody was incubated for $2 \mathrm{~h}$ at room temperature followed by membrane wash in TBST 3 times. Thereafter, ECL solution (A and B solutions were mixed 1:1) (Invitrogen) in appropriate volume was added onto the membrane in a dark room, and exposure was performed according to the fluorescent strength of the bands. Then, images were developed and fixed followed by scanning. Gray scanning was carried out using ImageJ software.

Enzyme-linked immunosorbent assay (ELISA). Peripheral blood was centrifuged at $8,500 \mathrm{x}$ g for $5 \mathrm{~min}$ at $4^{\circ} \mathrm{C}$. Supernatant was collected and added into the wells followed by rinsing using cleaning solution (400 $\mu \mathrm{l}$ in each well). Then, $50 \mu \mathrm{l}$ rabbit anti-BDNF (Cell Signaling Technology) was added into each well, and the plate was incubated on a vertical shaker for $1 \mathrm{~h}$. Next, the mix was discarded followed by rinsing 4 times. In each sampling well, $100 \mu 1$ serum sample prepared in advance was added. In the well for the standard, $100 \mu \mathrm{l}$ standard was added followed by $2 \mathrm{~h}$ of reaction on a shaker at room temperature followed by rinsing 4 times. In each well, $200 \mu \mathrm{l}$ of color development reagent was added for $30 \mathrm{~min}$ in a dark room at room temperature. A hundred microliters stopping solution was added in each well to terminate the reaction, and the plate was placed in a plate reader at $450 \mathrm{~nm}$ to detect the optical density. Accordingly, the expression level of BDNF was calculated. ELISA kit for BDNF (R\&D Systems, Minneapolis, MN, USA).

Psychological nursing care. Pediatric patients with depression usually suffer from loneliness, anxiety and other depressing
Table I. PCR primers.

Primer sequences

\begin{tabular}{ll}
\hline BDNF & F: 5'-ACTTGTACACTTCGGCCGGGTGAT-3' \\
& R: 5'-TTTGCGTCGCATCCAGGTAATT-3' \\
GAPDH & F: 5'-GGAGGATTACTGCGCTGGCTCCTA-3' \\
& R: 5'-GACTCATCGTACTCCTGCTTGCTG-3'
\end{tabular}

BDNF, brain-derived neurotrophic factor; GAPDH, glyceraldehyde 3-phosphate dehydrogenase; $F$, forward; $R$, reverse.

feelings, which can lead to the refusal of treatment. Thus, by understanding the psychological changes from all aspects and providing the necessary recognition education for patients, we inform the patients of the key role of the treatment to lessen the depression. In addition, we ameliorate the anxiety and resistance to treatment, relieve negative feelings such as pessimistic, lonely, and depressing feelings, correct the previous misconceptions and establish the right cognitive outlook, thereby prompting them to cooperate with the treatment. Regular nursing care was provided by a general ward nursing. Comprehensive nursing care included the general ward nursing care and psychological, bowel, and dietary nursing care, guidance for drug administration, and nursing care of exercise and family.

Nursing care of bowel and diet. Symptoms like anorexia usually appear in pediatric patients with depression. Thus, during the treatment, understanding the dietary habits and preferences of patients helped selecting the food that could satisfy the dietary habit and nutritious requirement of each patient.

Guidance of drug administration. As pediatric patients with depression usually resist drug administration, education for drug administration was critical during the treatment. This education could guarantee the correct pattern of drug administration, thereby ameliorating the side effect and increasing the compliance of drug administration.

Nursing care for exercise. Due to the psychological states in pediatric patients with depression, such as eccentricity and unwillingness to exercise, we stipulated a detailed work-out plan, and prompted the patients to fulfill their daily assignment, thereby encouraging the patients to have more outdoor exercise and raising their interest in exercise.

Familial nursing care. Care and encouragement from the family are critical to depressed child patients. Thus, nursing care was carried out by deploying the living condition that conformed to the interest of patients, and providing convenience and conformity for patients. Patients should experience the warmth from a close relationship with parents and family members. In addition, patients were encouraged to overcome the bad feelings and psychological obstacles bravely.

Hamilton depression (HAMD) assessment. We adopted the HAMD scale to evaluate depression. Scores were given to 
A

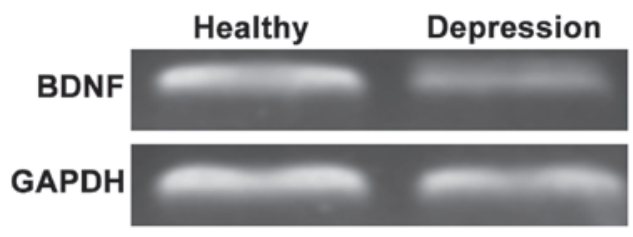

B

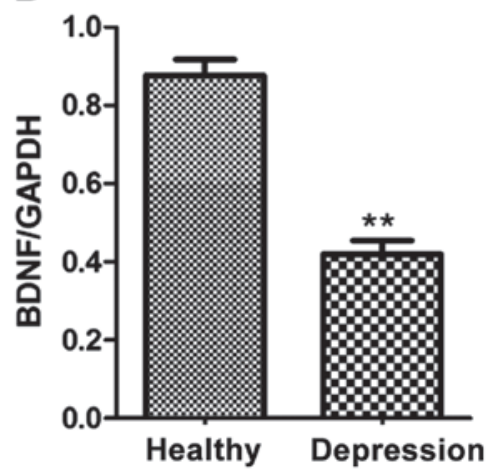

Figure 1. Detection of brain-derived neurotrophic factor (BDNF) mRNA by semi-quantitative PCR. (A) Agarose gel electrophoresis. (B) Statistical chart. Compared with healthy children, mRNA expression of BDNF in pediatric patients with depression is significantly decreased, ${ }^{* *}<<0.01$.

A

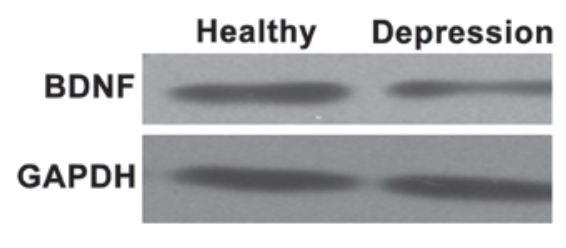

B

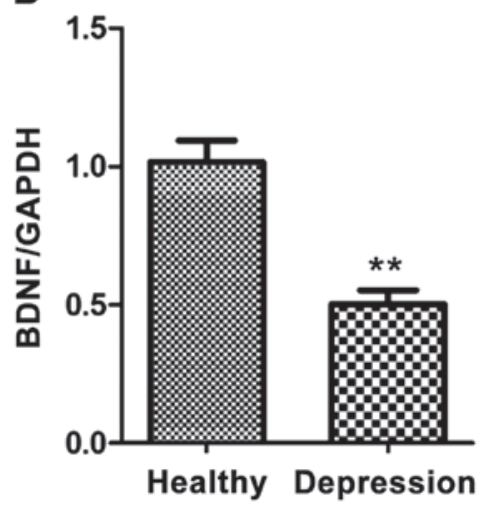

Figure 2. Detection of brain-derived neurotrophic factor (BDNF) protein by western blotting. (A) Histogram. (B) Statistical chart. Compared with healthy children, protein expression of BDNF in pediatric patients with depression is significantly decreased, ${ }^{* *} \mathrm{p}<0.01$.

each question and depression condition was divided into four categories. The severity of depression was evaluated according to the scores.

Compliance to treatment. Comprehensive evaluation was performed according to the cooperation of patients to treatment and compliance to drug administration. Complete compliance referred to patients that cooperated with the treatment and received medication strictly in accordance with the medical orders. Incomplete compliance referred to patients that cooperated with the treatment, but received the medication not strictly in accordance with the medical order. Non-compliance referred to patients that did not cooperate with the treatment or did not receive the medication in accordance with the orders. Finally, the cases of complete compliance and non-compliance were used to calculate the total compliance rate.

Quality of life. Through assessment of quality of life, we evaluated the progress of pediatric patients with depression. Scores were given to each question and the life quality was divided into 5 categories, with the high scores representing a better quality of life.

Statistical analysis. Data are presented as mean \pm standard deviation. SPSS 19.0 (Statistic Package for Social Science; SPSS, Inc., Chicago, IL, USA) was used for data processing.
Analysis by t-test was performed for comparison between two groups, and analysis of variance (ANOVA) for comparisons among groups. Through homogeneity test of variance, Bonferroni method was used for comparison between either two groups that had equal variance, Welch method for comparison between either two groups that had different variance, and Dunnett's T3 method for multiple comparisons. $\mathrm{p}<0.05$ suggested that the difference had statistical significance.

\section{Results}

$B D N F$ expression in serum in pediatric patients with depression. We first compared the levels of BDNF in blood from children with depression and healthy children. Semiquantitative PCR showed that the expression level of BDNF mRNA was lower in pediatric patients with depression compared with healthy children ( $<<0.01$ ) (Fig. 1). To confirm this reduction in $B D N F$ mRNA expression, we next examined the levels of BDNF protein. Compared with healthy children, BDNF protein level was low ( $<<0.01)$ (Fig. 2). Using the BDNF level in healthy children as standard, we selected 60 children with depression and low levels of BDNF. We found no statistically significant difference in the levels of BDNF within this group ( $p>0.05$ ). Then, these 60 patients were divided in two treatments: regular nursing $(\mathrm{n}=30)$ and comprehensive nursing $(n=30)$ groups. The two groups received the same medication, 


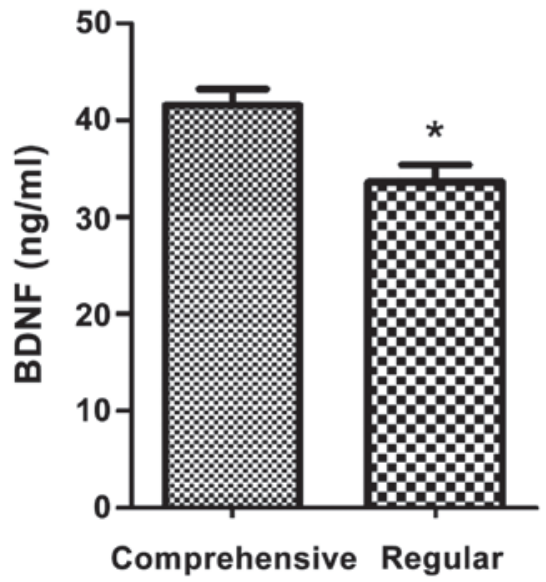

Figure 3. Detection of brain-derived neurotrophic factor (BDNF) protein expression by enzyme-linked immunosorbent assay (ELISA). After treatment, the protein expression of BDNF in pediatric patients in the comprehensive nursing group is significantly higher than that in the regular nursing group, ${ }^{*} \mathrm{p}<0.05$.

but patients in the comprehensive nursing care received additional physical, educational and emotional support. After 3 months of treatment, we examined BDNF expression by ELISA. Compared with the regular nursing group, patients subjected to comprehensive nursing care experienced an increase in BDNF expression with a statistically significant difference $(\mathrm{p}<0.05)$ (Fig. 3).

Depression measures using the HAMD scale. The HAMD scale was used to evaluate depression degree in three study groups: regular nursing $(n=30)$, the comprehensive nursing $(n=30)$, and healthy children $(n=50)$. Before the treatment, the HAMD score of pediatric patients with depression was significantly higher than that in the healthy children $(\mathrm{p}<0.01)$ (Table II). However, there was no statistically significant difference in the HAMD score between the regular nursing and the comprehensive nursing groups ( $>00.05)$ (Table II). After 3 months of treatment, patients that received comprehensive nursing care showed a significant decreased in depression scores $(\mathrm{p}<0.05)$ (Table III).

Compliance to treatment. Compliance to treatment of pediatric patients was evaluated in the comprehensive nursing and regular nursing groups. Before medication and nursing care, compliance was similar in both groups $(\mathrm{p}>0.05)$ (Table IV). After 3 months of treatment, compliance in patients that received comprehensive nursing care was significant higher than in those receiving regular nursing care $(\mathrm{p}<0.05)($ Table $\mathrm{V})$.

Quality of life. We used a questionnaire to evaluate quality of life in pediatric patients with depression. Before the treatment, the quality of life in the regular nursing and comprehensive nursing groups were significantly lower than that in healthy children $(\mathrm{p}<0.05)($ Table VI). However, there was no statistically significant difference in quality of life between the two nursing groups $(p>0.05)$ (Table VI). After 3 months of treatment, the quality of life in patients that received comprehensive nursing care was elevated compared with those receiving regular nursing care $(\mathrm{p}<0.05)$ (Table VII).
Table II. HAMD depression scores in pediatric patients before treatment.

\begin{tabular}{lcccc}
\hline Group & $\begin{array}{c}\text { Cognitive } \\
\text { dysfunction }\end{array}$ & Anxiety & Desperation & $\begin{array}{c}\text { Sleep } \\
\text { disorder }\end{array}$ \\
\hline $\begin{array}{l}\text { Regular } \\
\text { nursing }\end{array}$ & $21.87 \pm 1.61$ & $14.83 \pm 0.92$ & $12.61 \pm 1.38$ & $7.28 \pm 0.96$ \\
$\begin{array}{l}\text { Comprehensive } \\
\text { nursing }\end{array}$ & $22.11 \pm 1.53$ & $14.62 \pm 0.75$ & $13.15 \pm 1.56$ & $7.43 \pm 0.89$ \\
$\begin{array}{l}\text { Healthy } \\
\text { children }\end{array}$ & $5.23 \pm 1.15^{\mathrm{a}}$ & $3.25 \pm 0.72^{\mathrm{a}}$ & $2.82 \pm 0.85^{\mathrm{a}}$ & $1.46 \pm 0.37^{\mathrm{a}}$ \\
P-value & $<0.01$ & $<0.01$ & $<0.01$ & $<0.01$ \\
\hline
\end{tabular}

Compared with the healthy children, the depression in patients is more significant $\left({ }^{\mathrm{a}} \mathrm{p}<0.01\right)$. There is no significant difference in depression between the patients in regular nursing group and those in the comprehensive nursing group ( $\mathrm{p}>0.05)$. HAMD, Hamilton depression.

Table III. HAMD depression scores in pediatric patients before treatment and 3 months later.

\begin{tabular}{lcccc}
\hline Group & $\begin{array}{c}\text { Cognitive } \\
\text { dysfunction }\end{array}$ & Anxiety & Desperation & $\begin{array}{c}\text { Sleep } \\
\text { disorder }\end{array}$ \\
\hline Regular nursing & $8.53 \pm 0.76$ & $5.27 \pm 0.78$ & $4.26 \pm 0.65$ & $2.37 \pm 0.63$ \\
Comprehensive & $5.85 \pm 0.87$ & $3.65 \pm 0.86$ & $3.15 \pm 0.52$ & $1.86 \pm 0.52$ \\
nursing & $<0.05$ & $<0.05$ & $<0.05$ & $<0.05$ \\
P-value & & & & \\
\hline
\end{tabular}

HAMD, Hamilton depression.

\section{Discussion}

Depression is usually characterized by recurrence. Although some patients maintain a relatively normal mental state in remission after treatment, recurrence can be triggered by social and mental stress, and in extreme cases, patients will commit suicide $(11,12)$. BDNF is expressed in the cerebral cortex, hippocampus, thalamus, and cerebellum, with the highest expression corresponding to the hippocampus (13). Additionally, BDNF can nourish and protect central and peripheral neurons through regulating the metabolism and regeneration of neurons (14).

In this study, we used RT-PCR and western blot analysis to detect the expression level of BDNF in peripheral blood from pediatric patients with depression. Both assays showed that expression of BDNF in the patients was lower than in the control group. Clinical research has shown that BDNF levels in serum is equivalent to those in the brain tissues in depression patients, which is 100 times the level in plasma $(15,16)$. Thus, detecting BDNF expression in serum of peripheral blood effectively reflects the brain level of BDNF with the advantage of minimal invasion, simple procedure, and accurate results. According to one study, the expression level of BDNF in depression patients was significantly lower than that in the healthy population, and a lower BDNF expression 
Table IV. Compliance to treatment in patients before treatment.

\begin{tabular}{lccccc}
\hline Group & Cases (n) & Compliance compliance (n) & Incomplete (n) & Non-compliance & Compliance rate (\%) \\
\hline Regular nursing & 30 & 4 & 10 & 16 & 46.7 \\
Comprehensive nursing & 30 & 6 & 6 & 18 & 40.0 \\
P-value & & $>0.05$ & $>0.05$ & $>0.05$ & $>0.05$ \\
\hline
\end{tabular}

Table V. Compliance to treatment after nursing care.

\begin{tabular}{lccccc}
\hline Group & Cases & Compliance (n) & Incomplete compliance (n) & Non-compliance (n) & Compliance rate (\%) \\
\hline Regular nursing & 30 & 17 & 8 & 5 & 83.3 \\
Comprehensive nursing & 30 & 23 & $<$ & $<.05$ & $<0.0$ \\
P-value & & $<0.05$ & $<0.05$ & $<0.05$ \\
\hline
\end{tabular}

Table VI. Quality of life in pediatric patients and controls.

\begin{tabular}{lcccc}
\hline Item & Regular & $\begin{array}{c}\text { Comprehensive } \\
\text { nursing }\end{array}$ & $\begin{array}{c}\text { Healthy } \\
\text { children }\end{array}$ & P-value \\
\hline $\begin{array}{l}\text { Physical } \\
\text { performance }\end{array}$ & $43.29 \pm 5.26$ & $42.56 \pm 5.90$ & $63.38 \pm 6.32$ & $<0.01$ \\
$\begin{array}{l}\text { Psychological } \\
\text { performance }\end{array}$ & $36.57 \pm 4.81$ & $37.25 \pm 5.83$ & $52.65 \pm 5.27^{\mathrm{a}}$ & $<0.01$ \\
$\begin{array}{l}\text { Social function } \\
\begin{array}{l}\text { Physical living } \\
\text { condition }\end{array}\end{array}$ & $39.73 \pm 4.574 .29$ & $19.34 \pm 4.21$ & $31.67 \pm 4.53^{\mathrm{a}}$ & $<0.01$ \\
& & $38.59 \pm 4.65$ & $53.86 \pm 5.76^{\mathrm{a}}$ & $<0.01$ \\
\end{tabular}

Condition in pediatric patients with depression is more significant than that in the healthy children $\left({ }^{a} \mathrm{p}<0.01\right)$. There is no statistically significant difference in comparison of depression between the regular nursing group and the comprehensive nursing group $(\mathrm{p}>0.05)$.

Table VII. Quality of life in pediatric patients with depression after nursing care.

\begin{tabular}{lccc}
\hline Item & Regular nursing & Comprehensive nursing & P-value \\
\hline $\begin{array}{l}\text { Physical } \\
\text { performance }\end{array}$ & $53.87 \pm 5.31$ & $59.26 \pm 5.82$ & $<0.05$ \\
$\begin{array}{l}\text { Psychological } \\
\text { performance }\end{array}$ & $48.98 \pm 6.19$ & $51.65 \pm 5.27$ & $<0.05$ \\
$\begin{array}{l}\text { Social function } \\
\text { Material living } \\
\text { condition }\end{array}$ & $27.41 \pm 4.65$ & $30.59 \pm 4.49$ & $<0.05$ \\
\hline
\end{tabular}

represents the more severe depression (17). Severe depression and longer disease course, usually leads to neuronal apoptosis and pyknosis, causing a decrease in the intake of neurotrophic factors. Autopsy investigations showed that after medication of anti-depression drugs, the symptoms are significantly ameliorated and BDNF in the brain increased to normal levels (18). Therefore, the level of BDNF in the brain is closely correlated with the occurrence and development of depression. Here, we used the HAMD scale to evaluate depression. Pediatric patients with depression with low levels of BDNF were divided into regular and comprehensive nursing groups. Both groups received the same medical treatment, but after 3 months of nursing treatment, the recovery in the comprehensive nursing group was much higher than in the regular nursing group. In addition, we found that compliance and quality of life in the comprehensive nursing group were significantly superior to those in the regular nursing group. These results showed that comprehensive nursing care enhances the treatment for depression. Comprehensive nursing care, through psychological, exercise, and family interventions, can help the patients ameliorate the negative emotions, promote their interest, establish the correct life values, and open their mind, which are of great significance to the treatment of depression $(19,20)$.

In conclusion, the occurrence of depression is closely correlated with low levels of BDNF in serum. For the poor compliance and quality of life of pediatric patients with depression, we can carry out comprehensive nursing care to significantly improve their symptoms. These results can provide theoretical and practical guidance for the clinical treatment and nursing care of pediatric patients with depression.

\section{References}

1. Theorell T, Hammarström A, Aronsson G, Träskman Bendz L, Grape T, Hogstedt C, Marteinsdottir I, Skoog I and Hall C: A systematic review including meta-analysis of work environment and depressive symptoms. BMC Public Health 15: 738, 2015.

2. Kinser PA, Goehler LE and Taylor AG: How might yoga help depression? A neurobiological perspective. Explore (NY) 8: 118-126, 2012.

3. Pizzagalli DA: Depression, stress, and anhedonia: Toward a synthesis and integrated model. Annu Rev Clin Psychol 10: 393-423, 2014.

4. Fryers $\mathrm{T}$ and Brugha $\mathrm{T}$ : Childhood determinants of adult psychiatric disorder. Clin Pract Epidemol Ment Health 9: 1-50, 2013.

5. Hammerton G, Harold G, Thapar A and Thapar A: Depression and blood pressure in high-risk children and adolescents: An investigation using two longitudinal cohorts. BMJ Open 3: e003206, 2013.

6. Cummings CM, Caporino NE and Kendall PC: Comorbidity of anxiety and depression in children and adolescents: 20 years after. Psychol Bull 140: 816-845, 2014 
7. Fernandes BS, Molendijk ML, Köhler CA, Soares JC, Leite CM, Machado-Vieira R, Ribeiro TL, Silva JC, Sales PM, Quevedo J, et al: Peripheral brain-derived neurotrophic factor (BDNF) as a biomarker in bipolar disorder: A meta-analysis of 52 studies. BMC Med 13: 289, 2015.

8. Cheung T, Wong SY, Wong KY, Law LY, Ng K, Tong MT, Wong KY, Ng MY and Yip PS: Depression, anxiety and symptoms of stress among baccalaureate nursing students in Hong Kong: A cross-sectional study. Int J Environ Res Public Health 13: 779, 2016.

9. Smith M, Stolder ME, Jaggers B, Liu MF and Haedtke C: Depression training in nursing homes: Lessons learned from a pilot study. Issues Ment Health Nurs 34: 90-102, 2013.

10. Haugan G and Innstrand ST: The effect of self-transcendence on depression in cognitively intact nursing home patients. ISRN Psychiatry 2012: 301325, 2012.

11. Pérez-López FR, Pasupuleti V, Mezones-Holguin E, BenitesZapata VA, Thota P, Deshpande A and Hernandez AV: Effect of vitamin $\mathrm{D}$ supplementation during pregnancy on maternal and neonatal outcomes: A systematic review and meta-analysis of randomized controlled trials. Fertil Steril 103: 1278-88.e4, 2015.

12. Pence BW, O'Donnell JK and Gaynes BN: The depression treatment cascade in primary care: A public health perspective. Curr Psychiatry Rep 14: 328-335, 2012.

13. Fung J, Gelaye B, Zhong QY, Rondon MB, Sanchez SE, Barrios YV, Hevner K, Qiu C and Williams MA: Association of decreased serum brain-derived neurotrophic factor (BDNF) concentrations in early pregnancy with antepartum depression. BMC Psychiatry 15: 43, 2015.

14. Nomoto H, Baba H, Satomura E, Maeshima H, Takebayashi N, Namekawa Y, Suzuki T and Arai H: Serum brain-derived neurotrophic factor levels and personality traits in patients with major depression. BMC Psychiatry 15: 33, 2015.
15. Kreinin A, Lisson S, Nesher E, Schneider J, Bergman J, Farhat K, Farah J, Lejbkowicz F, Yadid G, Raskin L, et al: Blood BDNF level is gender specific in severe depression. PLoS One 10: e0127643, 2015.

16. Na KS, Won E, Kang J, Chang HS, Yoon HK, Tae WS, Kim YK, Lee MS, Joe SH, Kim H, et al: Brain-derived neurotrophic factor promoter methylation and cortical thickness in recurrent major depressive disorder. Sci Rep 6: 21089, 2016.

17. Legge RM, Sendi S, Cole JH, Cohen-Woods S, Costafreda SG, Simmons A, Farmer AE, Aitchison KJ, McGuffin P and $\mathrm{Fu} \mathrm{CH}$ : Modulatory effects of brain-derived neurotrophic factor Val66Met polymorphism on prefrontal regions in major depressive disorder. Br J Psychiatry 206: 379-384, 2015.

18. Elzinga BM, Molendijk ML, Oude Voshaar RC, Bus BA, Prickaerts J, Spinhoven P and Penninx BJ: The impact of childhood abuse and recent stress on serum brain-derived neurotrophic factor and the moderating role of BDNF Val66Met. Psychopharmacology (Berl) 214: 319-328, 2011.

19. Gerritsen DL1, Smalbrugge M, Teerenstra S, Leontjevas R, Adang EM, Vernooij-Dassen MJ, Derksen E and Koopmans RT: In case of depression: The evaluation of a care program to improve the detection and treatment of depression in nursing homes. Study Protocol. BMC Psychiatry 11: 91, 2011.

20. Park SC, Lee HY, Lee DW, Hahn SW, Park SH, Kim YJ, Choi JS, Lee HS, Lee SI, Na KS, et al: Knowledge and attitude of 851 nursing personnel toward depression in General Hospitals of Korea. J Korean Med Sci 30: 953-959, 2015. 